\title{
A GENERAL MEAN VALUE THEOREM
}

\author{
ZSOLT PÁLES
}

\begin{abstract}
In this note a general a Cauchy-type mean value theorem for the ratio of functional determinants is offered. It generalizes Cauchy's and Taylor's mean value theorems as well as other classical mean value theorems.
\end{abstract}

\section{INTRODUCTION}

The aim of the present note is to offer a unified approach to most of the mean value theorems known in elementary analysis.

Let $x_{1}, \ldots, x_{k}$ be arbitrary points in the real interval $[a, b]$. Then, one can uniquely determine a permutation $\pi$ of the set $\{1, \ldots, k\}, n \in \mathbb{N}, \xi_{1}<\cdots<\xi_{n}$ in $[a, b]$ and $k_{1}, \ldots, k_{n}$ in $\mathbb{N}$ with $k_{1}+\cdots+k_{n}=k$ such that

$$
\left(x_{\pi(1)}, \ldots, x_{\pi(k)}\right)=(\underbrace{\xi_{1}, \ldots, \xi_{1}}_{k_{1} \text { times }}, \ldots, \underbrace{\xi_{n}, \ldots, \xi_{n}}_{k_{n} \text { times }}) .
$$

If $w_{1}, \ldots, w_{m+k}:[a, b] \rightarrow \mathbb{R}$ is a system of $(k-1)$ times differentiable functions $(m>0)$, and $u_{1}, \ldots, u_{m+k} \in \mathbb{R}^{m}$ are vectors, then we define

$$
\begin{aligned}
& \mathcal{W}\left(\begin{array}{lll}
w_{1}, & \ldots, & w_{m+k} \\
u_{1}, & \ldots, & u_{m+k}
\end{array}\right)\left(x_{1}, \ldots, x_{k}\right)
\end{aligned}
$$

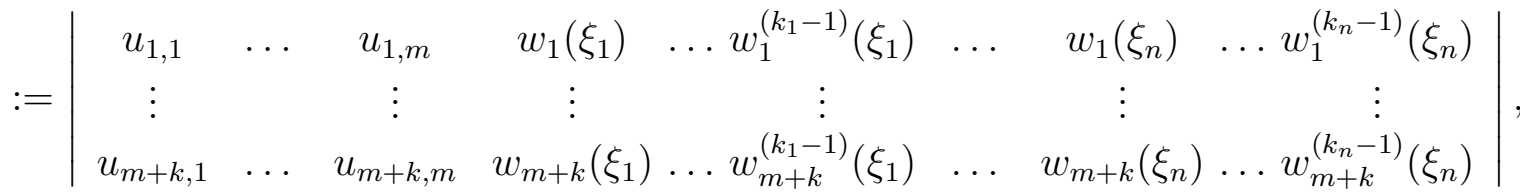

where the right hand side of this equation is an $(m+k) \times(m+k)$ determinant, $w^{(i)}$ stands for the $i$ th derivative of the function $w, u_{i, j}$ denotes the $j$ th coordinate of the vector $u_{i}$, and $\xi_{i}, k_{i}$ is determined by (11).

We also allow $m$ to take the value 0 , with the following notational conventions: $\mathbb{R}^{0}:=\{0\}$ and

$$
\begin{aligned}
\mathcal{W}\left(\begin{array}{ccc}
w_{1}, & \ldots, & w_{k} \\
u_{1}, & \ldots, & u_{k}
\end{array}\right)\left(x_{1}, \ldots, x_{k}\right) & :=\mathcal{W}\left(w_{1}, \ldots, w_{k}\right)\left(x_{1}, \ldots, x_{k}\right) \\
& :=\left|\begin{array}{ccccc}
w_{1}\left(\xi_{1}\right) \ldots & \ldots & w_{1}^{\left(k_{1}-1\right)}\left(\xi_{1}\right) & \ldots & w_{1}\left(\xi_{n}\right) \ldots w_{1}^{\left(k_{n}-1\right)}\left(\xi_{n}\right) \\
\vdots & \vdots & & \vdots & \vdots \\
w_{k}\left(\xi_{1}\right) \ldots & w_{k}^{\left(k_{1}-1\right)}\left(\xi_{1}\right) & \ldots & w_{k}\left(\xi_{n}\right) \ldots & \ldots \\
w_{k}^{\left(k_{n}-1\right)}\left(\xi_{n}\right)
\end{array}\right|,
\end{aligned}
$$

Date: July 21, 2015.

1991 Mathematics Subject Classification. Primary 26A51, $26 B 25$.

Key words and phrases. Mean value theorem, Wronski determinant, Quasi differential operators.

This research has been supported by the Hungarian National Research Science Foundation (OTKA) Grant K111651. 
Observe that if here $x_{1}=\cdots=x_{k}=\xi$, then the above definition reduces to

$$
\mathcal{W}\left(w_{1}, \ldots, w_{k}\right)(\xi, \ldots, \xi)=\left|\begin{array}{ccc}
w_{1}(\xi) & \cdots & w_{1}^{(k-1)}(\xi) \\
\vdots & \ddots & \vdots \\
w_{k}(\xi) & \cdots & w_{k}^{(k-1)}(\xi)
\end{array}\right|,
$$

which is known as the Wronski determinant of the system $w_{1}, \ldots, w_{k}$.

The class of functions $f:[a, b] \rightarrow \mathbb{R}$ that are $k-1$ times continuously differentiable on $[a, b]$ and $k$ times differentiable on the open interval $] a, b\left[\right.$ will be denoted by $D^{k}([a, b])$.

Now we are able to formulate the main result of this paper.

Theorem 1. Let $1 \leq k, 0 \leq m$ be integers and $u_{1}, \ldots, u_{m+k} \in \mathbb{R}^{m}$ such that (if $0<m$ then) $u_{1}, \ldots, u_{m}$ are linearly independent, i.e.,

$$
\mathcal{V}_{0}:=\left|\begin{array}{ccc}
u_{1,1} & \cdots & u_{1, m} \\
\vdots & \ddots & \vdots \\
u_{m, 1} & \cdots & u_{m, m}
\end{array}\right| \neq 0
$$

In addition, let $w_{1}, \ldots, w_{m+k} \in D^{k}([a, b])$ be a system of functions satisfying

$$
\mathcal{V}_{n}(\xi):=\mathcal{W}\left(\begin{array}{lll}
w_{1}, & \ldots, & w_{m+n} \\
u_{1}, & \ldots, & u_{m+n}
\end{array}\right)(\underbrace{\xi, \ldots, \xi}_{n \text { times }}) \neq 0
$$

for all $\xi \in[a, b]$ and $n=1, \ldots, k$. Then, for all nonidentical points $x_{1}, \ldots, x_{k+1} \in[a, b]$, vectors $p, q \in \mathbb{R}^{m}$ and functions $f, g \in D^{k}([a, b])$, there exists an intermediate point $\left.\xi \in\right] \min x_{i}$, $\max x_{i}[$ such that

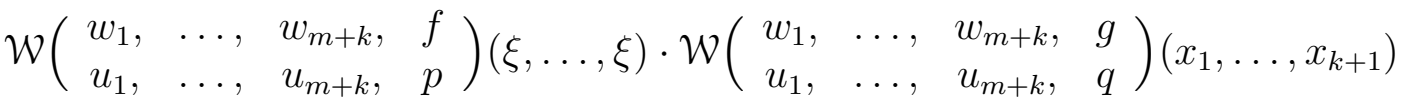

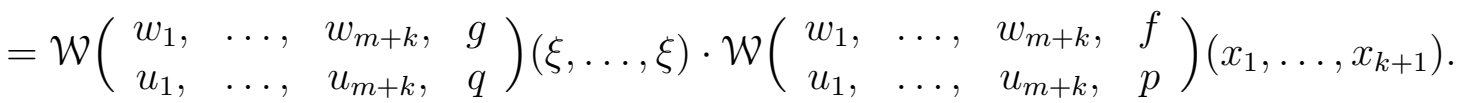

The proof of this theorem is given in the next section. Now we list some of its consequences. Corollary 1. (Cauchy's Mean Value Theorem.) Let $f, g \in D^{1}[a, b]$ Then there exists $\left.\xi \in\right] a, b[$ such that

$$
f^{\prime}(\xi)(g(a)-g(b))=g^{\prime}(\xi)(f(a)-f(b)) .
$$

Proof. Let $k=1, m=0, w_{1} \equiv 1$ and $x_{1}=a, x_{2}=b$ in Theorem 1 . Then the statement follows immediately from (4).

Corollary 2. (Taylor's Mean Value Theorem.) Let $f \in D^{k}([a, b])$. Then, for all $\left.\left.x \in\right] a, b\right]$, there exists $\xi \in] a, b[$ such that

$$
f(x)=f(a)+f^{\prime}(a)(x-a)+\cdots+\frac{f^{(k-1)}(a)}{(k-1) !}(x-a)^{k-1}+\frac{f^{(k)}(\xi)}{k !}(x-a)^{k} .
$$

Proof. Let $m=0$,

$$
w_{i}(x)=\frac{(x-a)^{i-1}}{(i-1) !} \quad \text { for } \quad i=1, \ldots, k \quad \text { and } \quad g(x)=\frac{(x-a)^{k}}{k !} .
$$


Then, for all $\xi \in[a, b]$,

$$
\mathcal{W}\left(w_{1}\right)(\xi)=\cdots=\mathcal{W}\left(w_{1}, \ldots, w_{k}\right)(\xi, \ldots, \xi)=1,
$$

therefore, (2) and (3) are satisfied. Thus, taking $x_{1}=\cdots=x_{k}=a$ and $x_{k+1}=x$ in Theorem 1 , we obtain that there exists $\xi \in] a, x[$ satisfying

$$
\begin{aligned}
\mathcal{W}\left(w_{1}, \ldots, w_{k}, f\right)(\xi, \ldots, \xi, \xi) \cdot \mathcal{W}\left(w_{1}, \ldots, w_{k}, g\right)(a, \ldots, a, x) \\
=\mathcal{W}\left(w_{1}, \ldots, w_{k}, g\right)(\xi, \ldots, \xi, \xi) \cdot \mathcal{W}\left(w_{1}, \ldots, w_{k}, f\right)(a, \ldots, a, x) .
\end{aligned}
$$

A simple computation yields that

$$
\begin{gathered}
\mathcal{W}\left(w_{1}, \ldots, w_{k}, f\right)(a, \ldots, a, x)=f(x)-f(a)-f^{\prime}(a)(x-a)-\cdots-\frac{f^{(k-1)}(a)}{(k-1) !}(x-a)^{k-1}, \\
\mathcal{W}\left(w_{1}, \ldots, w_{k}, g\right)(a, \ldots, a, x)=\frac{(x-a)^{k}}{k !}
\end{gathered}
$$

furthermore

$$
\mathcal{W}\left(w_{1}, \ldots, w_{k}, f\right)(\xi, \ldots, \xi, \xi)=f^{(k)}(\xi) \quad \text { and } \quad \mathcal{W}\left(w_{1}, \ldots, w_{k}, g\right)(\xi, \ldots, \xi, \xi)=1 .
$$

Thus, Taylor's theorem follows from (5) at once.

Let $w_{1}(x)=1, \ldots, w_{k}(x)=x^{k-1}, w_{k+1}=x^{k}$ for $x \in[a, b]$ and let $a \leq x_{1} \leq \cdots \leq x_{k+1} \leq b$ with $a<x_{k+1}$ and $x_{1}<b$. Then the ratio

$$
\left[x_{1}, \ldots, x_{k+1}\right] f:=\frac{\mathcal{W}\left(w_{1}, \ldots, w_{k}, f\right)\left(x_{1}, \ldots, x_{k}, x_{k+1}\right)}{\mathcal{W}\left(w_{1}, \ldots, w_{k}, w_{k+1}\right)\left(x_{1}, \ldots, x_{k}, x_{k+1}\right)}
$$

is called the $k$ th order divided difference of $f \in D^{k}([a, b])$ over the points $x_{1}, \ldots, x_{k+1}$ (c.f. [Sch81, p. 45]). Divided differences are usually defined in an inductive way in the literature, see e.g. [AH79, Sect. 3.17] and [HA38, Sect. 2.3]. The proof of the equivalence of the above definition to the usual one can be found in [Sch81, Theorem 2.51, p.47].

Concerning divided differences, the following result is well known (c.f. [AH79, p. 274] and [Sch81, (2.93)]).

Corollary 3. Let $f \in D^{k}([a, b])$ and $a \leq x_{1} \leq \cdots \leq x_{k+1} \leq b$ with $x_{1}<x_{k+1}$. Then there exists $\xi \in] x_{1}, x_{k+1}[$ such that

$$
\left[x_{1}, \ldots, x_{k+1}\right] f=\frac{f^{(k)}(\xi)}{k !} .
$$

Proof. Apply Theorem 1 when $m=0$ with the function $g(x)=w_{k+1}(x)=x^{k}$. Then we find that there exists $\xi \in] x_{1}, x_{k+1}[$ such that

$$
\left[x_{1}, \ldots, x_{k+1}\right] f=[\underbrace{\xi, \ldots, \xi}_{k+1 \text { times }}] f=\frac{f^{(k)}(\xi)}{k !} .
$$

Thus (6) is proved.

The following result, called Cauchy Mean Value Theorem, is due to Rätz and Russel [RR87]. 
Corollary 4. Let $f, g \in D^{k}([a, b])$ such that $g^{(k)}(\xi) \neq 0$ for $\left.\xi \in\right] a, b\left[\right.$ and let $a \leq x_{1} \leq \cdots \leq$ $x_{k+1} \leq b$ with $x_{1}<x_{k+1}$. Then there exists $\left.\xi \in\right] x_{1}, x_{k+1}[$ such that

$$
\frac{\left[x_{1}, \ldots, x_{k+1}\right] f}{\left[x_{1}, \ldots, x_{k+1}\right] g}=\frac{f^{(k)}(\xi)}{g^{(k)}(\xi)} .
$$

Proof. Applying Corollary 3 for the function $g$ first, we can observe that

$$
\left[x_{1}, \ldots, x_{k+1}\right] g \neq 0 .
$$

Hence the left hand side of (7) exists. Clearly,

$$
\frac{\left[x_{1}, \ldots, x_{k+1}\right] f}{\left[x_{1}, \ldots, x_{k+1}\right] g}=\frac{\mathcal{W}\left(w_{1}, \ldots, w_{k}, f\right)\left(x_{1}, \ldots, x_{k}, x_{k+1}\right)}{\mathcal{W}\left(w_{1}, \ldots, w_{k}, g\right)\left(x_{1}, \ldots, x_{k}, x_{k+1}\right)}
$$

Therefore, by Theorem 1 , there exists $\xi \in] x_{1}, x_{k+1}[$ such that

$$
\frac{\left[x_{1}, \ldots, x_{k+1}\right] f}{\left[x_{1}, \ldots, x_{k+1}\right] g}=\frac{\mathcal{W}\left(w_{1}, \ldots, w_{k}, f\right)(\xi, \ldots, \xi, \xi)}{\mathcal{W}\left(w_{1}, \ldots, w_{k}, g\right)(\xi, \ldots, \xi, \xi)}=\frac{f^{(k)}(\xi)}{g^{(k)}(\xi)},
$$

whence (7) follows.

\section{Proof of the MAIN RESUlt}

In the proof of Theorem 1, we shall need the following notion: A function $f \in D^{k}([a, b])$ vanishes $k+1$ times in $[a, b]$ if there exist points $x_{1}<\cdots<x_{n}$ in $[a, b]$ with $x_{1}<b, a<x_{n}$ and natural numbers $k_{1}, \ldots, k_{n}$ with $k_{1}+\cdots+k_{n}=k+1$ such that

$$
f^{(j)}\left(x_{i}\right)=0 \quad \text { for } \quad j=0, \ldots, k_{i}-1, i=1, \ldots, n .
$$

For instance, the function $f(x)=x(x-1)$ vanishes twice in $[0,1]$. However the function $f(x)=x^{2}$ does not vanish twice in $[0,1]$, but it does in $[-1,1]$, (that is, all the zeroes of $f$ should not be concentrated at the endpoints of the interval).

We recall the following lemmas from Pal94] and, for readers convenience, we also provide their proofs.

Lemma 1. If $f, g \in D^{k}([a, b])$ and $f$ vanishes $k+1$ times in $[a, b]$, then $f g$ also vanishes $k+1$ times in $[a, b]$.

Proof. By the assumption, there are $x_{1}<\cdots<x_{n}$ in $[a, b]$ with $x_{1}<b, a<x_{n}$ and $k_{1}, \ldots, k_{n} \in$ $\mathbb{N}$ with $k_{1}+\cdots+k_{n}=k+1$ such that (8) holds. Then, using Leibniz's Product Rule, one can check that

$$
(f g)^{(j)}\left(x_{i}\right)=0 \quad \text { for } \quad j=0, \ldots, k_{i}-1, i=1, \ldots, n .
$$

Thus $f g$ also vanishes $k+1$ times in $[a, b]$.

Lemma 2. If $f \in D^{k}([a, b])$ vanishes $k+1$ times in $[a, b]$, then $f^{\prime}$ vanishes $k$ times in $[a, b]$.

Proof. We have (8) for some $x_{1}<\cdots<x_{n}$ with $x_{1}<b, a<x_{n}$ and $k_{1}, \ldots, k_{n} \in \mathbb{N}$ with $k_{1}+\cdots+k_{n}=k+1$. If $n=1$, then there is nothing to prove. Otherwise, by Rolle's Mean Value Theorem, there exist $x_{i}<\xi_{i}<x_{i+1}$ such that

$$
f^{\prime}\left(\xi_{i}\right)=0 \quad \text { for } \quad i=1, \ldots, n-1 .
$$

These equalities combined with (8) yield that $f^{\prime}$ vanishes $k$ times on $[a, b]$. 
The following lemma generalizes [Pal94, Lemma 3]. The result obtained therein corresponds the case $m=0$ below.

Lemma 3. Let $1 \leq k, 0 \leq m$ be integers and $u_{1}, \ldots, u_{m+k} \in \mathbb{R}^{m}$ such that (2) holds (if $m>0)$. Let $w_{1}, \ldots, w_{m} \in D^{k}([a, b])$ be a system of functions satisfying (3) for all $\xi \in[a, b]$. For $f \in D^{k}([a, b])$, define the following operators

$$
\mathbb{W}_{n}(f)(\xi):=\mathcal{W}\left(\begin{array}{llll}
w_{1}, & \ldots, & w_{m+n}, & f \\
u_{1}, & \ldots, & u_{m+n}, & 0
\end{array}\right)(\underbrace{\xi, \ldots, \xi}_{n+1 \text { times }}, \quad n=0, \ldots, k .
$$

where $\xi \in[a, b]$ if $n<k$, and $\xi \in] a, b[$ if $n=k$. Then the following recursive formula

$$
\mathbb{W}_{n}(f)(\xi)=\frac{d}{d \xi}\left(\frac{\mathbb{W}_{n-1}(f)(\xi)}{\mathcal{V}_{n}(\xi)}\right) \cdot \frac{\left[\mathcal{V}_{n}(\xi)\right]^{2}}{\mathcal{V}_{n-1}(\xi)}
$$

holds for all $\xi \in[a, b]$ if $1 \leq n<k$, and for all $\xi \in] a, b\left[\right.$ if $n=k$. (Here $\mathcal{V}_{0}$ and $\mathcal{V}_{n}(1 \leq n \leq k)$ are defined in (21) and in (3), respectively. In the case $m=0$ we set $\mathcal{V}_{0}=0$.)

Proof. The argument described below works for $m \neq 0$. The $m=0$ case is completely analogous, therefore omitted.

The vectors $u_{1}, \ldots, u_{m}$ are linearly independent in $\mathbb{R}^{m}$, hence they form a basis of $\mathbb{R}^{m}$. Thus, we can find real numbers $\gamma_{1, n}, \ldots, \gamma_{m, n}$ such that, for $n=1, \ldots, k$,

$$
-u_{m+n}=\gamma_{1, n} u_{1}+\cdots+\gamma_{m, n} u_{m}
$$

Then define the functions $v_{n}:[a, b] \rightarrow \mathbb{R}$ by

$$
v_{n}:=w_{m+n}+\gamma_{1, n} w_{1}+\cdots+\gamma_{m, n} w_{m} .
$$

Now we show that the functions $v_{1}, \ldots, v_{n}$ form a linearly independent system of solutions of the equation

$$
\left.\mathbb{W}_{n}(f)(\xi)=0, \quad \xi \in\right] a, b[
$$

which is an $n$th order homogeneous linear differential equation for the unknown function $f$.

To see this, we first compute $\mathbb{W}_{n}\left(v_{j}\right)$ for any $1 \leq j \leq k$ and $0 \leq n \leq k$. Multiplying the first $m$ rows of the determinant $\mathbb{W}_{n}\left(v_{j}\right)$ by $\gamma_{1, j}, \ldots, \gamma_{m, j}$, respectively, subtracting their sum from the last row, then using (10), we get

$$
\begin{aligned}
& \mathbb{W}_{n}\left(v_{j}\right)(\xi)=\mathcal{W}\left(\begin{array}{cccc}
w_{1}, & \ldots, & w_{m+n}, & w_{m+j}+\sum_{i=1}^{m} \gamma_{i, j} w_{i} \\
u_{1}, & \ldots, & u_{m+n}, & 0
\end{array}\right)(\xi, \ldots, \xi) \\
& =\mathcal{W}\left(\begin{array}{cccc}
w_{1}, & \ldots, & w_{m+n}, & w_{m+j} \\
u_{1}, & \ldots, & u_{m+n}, & -\sum_{i=1}^{m} \gamma_{i, j} u_{i}
\end{array}\right)(\xi, \ldots, \xi) \\
& =\mathcal{W}\left(\begin{array}{cccc}
w_{1}, & \ldots, & w_{m+n}, & w_{m+j} \\
u_{1}, & \ldots, & u_{m+n}, & u_{m+j}
\end{array}\right)(\xi, \ldots, \xi)=0
\end{aligned}
$$

If $j \leq n$, then this formula results $\mathbb{W}_{n}\left(v_{j}\right)=0$. On the other hand, with $j=n+1$, we have $\mathbb{W}_{n}\left(v_{n+1}\right)=\mathcal{V}_{n+1}$.

The function $v_{1}$ cannot be identically zero because $\mathbb{W}_{0}\left(v_{1}\right)=\mathcal{V}_{1} \neq 0$. Hence $\left\{v_{1}\right\}$ is a linearly independent set of solutions of $\mathbb{W}_{1}(f)=0$. Assume now that $v_{1}, \ldots, v_{n}$ form a linearly independent system of solutions of $\mathbb{W}_{n}(f)=0$. The function $v_{n+1}$ is not a solution of this 
equation, hence, it cannot be a linear combination of $v_{1}, \ldots, v_{n}$. Thus, $v_{1}, \ldots, v_{n+1}$ is also a linearly independent system.

Temporarily, denote the operator defined by the right hand side of (9) by $\mathbb{W}_{n}^{*}(f)$. It is clear that $\mathbb{W}_{n}^{*}(f)$ is also an $n$ th-order linear differential operator of $f$. We show that $v_{1}, \ldots, v_{n}$ also solves the equation $\mathbb{W}_{n}^{*}(f)=0$. This is obvious if $f=v_{1}, \ldots, v_{n-1}$ (since these functions are solutions of the equation $\left.\mathbb{W}_{n-1}(f)=0\right)$. On the other hand

$$
\mathbb{W}_{n}^{*}\left(v_{n}\right)(\xi)=\frac{d}{d \xi}\left(\frac{\mathbb{W}_{n-1}\left(v_{n}\right)(\xi)}{\mathcal{V}_{n}(\xi)}\right) \cdot \frac{\left[\mathcal{V}_{n}(\xi)\right]^{2}}{\mathcal{V}_{n-1}(\xi)}=\frac{d}{d \xi}\left(\frac{\mathcal{V}_{n}(\xi)}{\mathcal{V}_{n}(\xi)}\right) \cdot \frac{\left[\mathcal{V}_{n}(\xi)\right]^{2}}{\mathcal{V}_{n-1}(\xi)}=0
$$

Observe that the coefficients of $f^{(n)}$ in $\mathbb{W}_{n}(f)$ and $\mathbb{W}_{n}^{*}(f)$ are equal to $\mathcal{V}_{n}$ which does not vanish anywhere in $[a, b]$. Therefore, having the same solution space, these two operators have to coincide for all $1 \leq n \leq k$.

Lemma 4. Let $1 \leq k, 0 \leq m$ be integers and $u_{1}, \ldots, u_{m+k} \in \mathbb{R}^{m}$ such that (2) holds (if $m>0$ ). Let $w_{1}, \ldots, w_{m} \in D^{k}([a, b])$ be a system of functions satisfying (3) for all $\xi \in[a, b]$. Assume that the function $f \in D^{k}([a, b])$ vanishes $k+1$ times in $[a, b]$. Then, for each $0 \leq n \leq k$, the function $\mathbb{W}_{n}(f)$ defined in Lemma 3 vanishes $k+1-n$ times in $[a, b]$.

Proof. We prove by induction. If $n=0$, then $\mathbb{W}_{0}(f)=\mathcal{V}_{0} f$, hence, in this case, there is nothing to prove. Let $n \geq 1$ and assume that $\mathbb{W}_{n-1}(f)$ vanishes $k+1-(n-1)$ times. Then, applying Lemma 1 and Lemma 2, one sees that the function

$$
\frac{d}{d \xi}\left(\frac{\mathbb{W}_{n-1}(f)(\xi)}{\mathcal{V}_{n}(\xi)}\right) \cdot \frac{\left[\mathcal{V}_{n}(\xi)\right]^{2}}{\mathcal{V}_{n-1}(\xi)} \quad(\xi \in[a, b])
$$

vanishes $k+1-(n-1)-1=k+1-n$ times. Therefore, due to the recursive formula established in Lemma $3, \mathbb{W}_{n}(f)$ vanishes $k+1-n$ times.

Now we are ready to prove the main result of the paper.

Proof of Theorem 1. Let $x_{1} \leq \cdots \leq x_{k+1}$ be in $[a, b]$ with $\min x_{i}<\max x_{i}$. Then there exist a permutation $\pi$ of $\left\{1, \ldots, x_{k+1}\right\}, n \in \mathbb{N}, \xi_{1}<\cdots<\xi_{n}$ in $[a, b]$ and $k_{1}, \ldots, k_{n} \in \mathbb{N}$ with $k_{1}+\cdots+k_{n}=k+1$ such that

$$
\left(x_{\pi(1)}, \ldots, x_{\pi(k+1)}\right)=(\underbrace{\xi_{1}, \ldots, \xi_{1}}_{k_{1} \text { times }}, \ldots, \underbrace{\xi_{n}, \ldots, \xi_{n}}_{k_{n} \text { times }})
$$

holds. Define the function $F:[a, b] \rightarrow \mathbb{R}$ by

$$
F(x):=\left|\begin{array}{ccccccccc}
u_{1,1} & \ldots & u_{1, m} & w_{1}\left(\xi_{1}\right) & \ldots & w_{1}^{\left(k_{1}-1\right)}\left(\xi_{1}\right) & \ldots & w_{1}^{\left(k_{n}-1\right)}\left(\xi_{n}\right) & w_{1}(x) \\
\vdots & & \vdots & \vdots & & \vdots & & \vdots & \vdots \\
u_{m+k, 1} & \ldots & u_{m+k, m} & w_{m+k}\left(\xi_{1}\right) & \ldots & w_{m+k}^{\left(k_{1}-1\right)}\left(\xi_{1}\right) & \ldots & w_{m+k}^{\left(k_{n}-1\right)}\left(\xi_{n}\right) & w_{m+k}(x) \\
p_{1} & \ldots & p_{m} & f\left(\xi_{1}\right) & \ldots & f^{\left(k_{1}-1\right)}\left(\xi_{1}\right) & \ldots & f^{\left(k_{n}-1\right)}\left(\xi_{n}\right) & f(x) \\
q_{1} & \ldots & q_{m} & g\left(\xi_{1}\right) & \ldots & g^{\left(k_{1}-1\right)}\left(\xi_{1}\right) & \ldots & g^{\left(k_{n}-1\right)}\left(\xi_{n}\right) & g(x)
\end{array}\right| .
$$

It is obvious at once that

$$
F^{(j)}\left(\xi_{i}\right)=0 \quad \text { for } \quad j=0, \ldots, k_{i}-1, i=1, \ldots, n,
$$


therefore $F$ vanishes $k+1$ times in $[a, b]$. Thus, by Lemma 4 , there exists $\xi \in] a, b[$ such that

$$
\mathbb{W}_{k}(F)(\xi)=\mathcal{W}\left(\begin{array}{llll}
w_{1}, & \ldots, & w_{m+k}, & F \\
u_{1}, & \ldots, & u_{m+k}, & 0
\end{array}\right)(\underbrace{\xi, \ldots, \xi}_{k+1 \text { times }})=0
$$

Now determine the contstants $\gamma_{i, n}$ such that they satisfy (10) and define $v_{1}, \ldots, v_{k}$ by (11). Similarly, choose $\alpha_{1}, \ldots, \alpha_{m}$ and $\beta_{1}, \ldots, \beta_{m}$ such that

$$
-p=\alpha_{1} u_{1}+\cdots+\alpha_{m} u_{m} \quad \text { and } \quad-q=\beta_{1} u_{1}+\cdots+\beta_{m} u_{m}
$$

and define

$$
\phi=f+\alpha_{1} w_{1}+\cdots+\alpha_{m} w_{m} \quad \text { and } \quad \psi=g+\beta_{1} w_{1}+\cdots+\beta_{m} w_{m} .
$$

Using these choices of the constants, add linear combination of the first $m$ rows of $F$ to the rest of the rows to obtain

$$
\begin{aligned}
& F(x):=\left|\begin{array}{ccccccccc}
u_{1,1} & \ldots & u_{1, m} & w_{1}\left(\xi_{1}\right) & \ldots & w_{1}^{\left(k_{1}-1\right)}\left(\xi_{1}\right) & \ldots & w_{1}^{\left(k_{n}-1\right)}\left(\xi_{n}\right) & w_{1}(x) \\
\vdots & & \vdots & \vdots & & \vdots & & \vdots & \vdots \\
u_{m, 1} & \ldots & u_{m, m} & w_{m}\left(\xi_{1}\right) & \ldots & w_{m}^{\left(k_{1}-1\right)}\left(\xi_{1}\right) & \ldots & w_{m}^{\left(k_{n}-1\right)}\left(\xi_{n}\right) & w_{m}(x) \\
0 & \ldots & 0 & v_{1}\left(\xi_{1}\right) & \ldots & v_{1}^{\left(k_{1}-1\right)}\left(\xi_{1}\right) & \ldots & v_{1}^{\left(k_{n}-1\right)}\left(\xi_{n}\right) & v_{1}(x) \\
\vdots & & \vdots & \vdots & & \vdots & & \vdots & \vdots \\
0 & \ldots & 0 & v_{k}\left(\xi_{1}\right) & \ldots & v_{k}^{\left(k_{1}-1\right)}\left(\xi_{1}\right) & \ldots & v_{k}^{\left(k_{n}-1\right)}\left(\xi_{n}\right) & v_{k}(x) \\
0 & \ldots & 0 & \phi\left(\xi_{1}\right) & \ldots & \phi^{\left(k_{1}-1\right)}\left(\xi_{1}\right) & \ldots & \phi^{\left(k_{n}-1\right)}\left(\xi_{n}\right) & \phi(x) \\
0 & \ldots & 0 & \psi\left(\xi_{1}\right) & \ldots & \psi^{\left(k_{1}-1\right)}\left(\xi_{1}\right) & \ldots & \psi^{\left(k_{n}-1\right)}\left(\xi_{n}\right) & \psi(x)
\end{array}\right| \\
& =V_{0} \cdot\left|\begin{array}{cccccc}
v_{1}\left(\xi_{1}\right) & \ldots & v_{1}^{\left(k_{1}-1\right)}\left(\xi_{1}\right) & \ldots & v_{1}^{\left(k_{n}-1\right)}\left(\xi_{n}\right) & v_{1}(x) \\
\vdots & & \vdots & & \vdots & \vdots \\
v_{k}\left(\xi_{1}\right) & \ldots & v_{k}^{\left(k_{1}-1\right)}\left(\xi_{1}\right) & \ldots & v_{k}^{\left(k_{n}-1\right)}\left(\xi_{n}\right) & v_{k}(x) \\
\phi\left(\xi_{1}\right) & \ldots & \phi^{\left(k_{1}-1\right)}\left(\xi_{1}\right) & \ldots & \phi^{\left(k_{n}-1\right)}\left(\xi_{n}\right) & \phi(x) \\
\psi\left(\xi_{1}\right) & \ldots & \psi^{\left(k_{1}-1\right)}\left(\xi_{1}\right) & \ldots & \psi^{\left(k_{n}-1\right)}\left(\xi_{n}\right) & \psi(x)
\end{array}\right| .
\end{aligned}
$$

Expanding by the last column, we get

$$
F(x)=\sum_{i=1}^{k} C_{i} \cdot v_{i}(x)-A \phi(x)+B \psi(x),
$$

where $A, B, C_{i}$ are the values of the corresponding subdeterminants. Substituting the above form of $F$ into (14), and using that $\mathbb{W}_{k}\left(v_{i}\right)=0$, we get that

$$
A \cdot \mathbb{W}_{k}(\phi)(\xi)=B \cdot \mathbb{W}_{k}(\psi)(\xi)
$$

In the rest of the proof we show that (17) reduces to (4). 
Indeed, adding a certain linear combination to the last row of $\mathbb{W}_{k}$, we get

$$
\begin{aligned}
& \mathbb{W}_{k}(\phi)(\xi)=\mathcal{W}\left(\begin{array}{cccc}
w_{1}, & \ldots, & w_{m+k}, & f+\sum_{i=1}^{m} \alpha_{i} w_{i} \\
u_{1}, & \ldots, & u_{m+k}, & 0
\end{array}\right)(\xi, \ldots, \xi) \\
& =\mathcal{W}\left(\begin{array}{cccc}
w_{1}, & \ldots, & w_{m+k}, & f \\
u_{1}, & \ldots, & u_{m+k}, & -\sum_{i=1}^{m} \alpha_{i} u_{i}
\end{array}\right)(\xi, \ldots, \xi) \\
& =\mathcal{W}\left(\begin{array}{cccc}
w_{1}, & \ldots, & w_{m+k}, & f \\
u_{1}, & \ldots, & u_{m+k}, & p
\end{array}\right)(\xi, \ldots, \xi) .
\end{aligned}
$$

For the constant $A$, due to its origin, we have

$$
A=V_{0} \cdot\left|\begin{array}{ccccc}
v_{1}\left(\xi_{1}\right) & \ldots & v_{1}^{\left(k_{1}-1\right)}\left(\xi_{1}\right) & \ldots & v_{1}^{\left(k_{n}-1\right)}\left(\xi_{n}\right) \\
\vdots & & \vdots & & \vdots \\
v_{k}\left(\xi_{1}\right) & \ldots & v_{k}^{\left(k_{1}-1\right)}\left(\xi_{1}\right) & \ldots & v_{k}^{\left(k_{n}-1\right)}\left(\xi_{n}\right) \\
\psi\left(\xi_{1}\right) & \ldots & \psi^{\left(k_{1}-1\right)}\left(\xi_{1}\right) & \ldots & \psi^{\left(k_{n}-1\right)}\left(\xi_{n}\right)
\end{array}\right|
$$

Now, using an argument similar to that applied in the computation of $F$, one can get that

$$
\begin{aligned}
& A=\left|\begin{array}{cccccccc}
u_{1,1} & \ldots & u_{1, m} & w_{1}\left(\xi_{1}\right) & \ldots & w_{1}^{\left(k_{1}-1\right)}\left(\xi_{1}\right) & \ldots & w_{1}^{\left(k_{n}-1\right)}\left(\xi_{n}\right) \\
\vdots & & \vdots & \vdots & & \vdots & & \vdots \\
u_{m, 1} & \ldots & u_{m, m} & w_{m}\left(\xi_{1}\right) & \ldots & w_{m}^{\left(k_{1}-1\right)}\left(\xi_{1}\right) & \ldots & w_{m}^{\left(k_{n}-1\right)}\left(\xi_{n}\right) \\
0 & \ldots & 0 & v_{1}\left(\xi_{1}\right) & \ldots & v_{1}^{\left(k_{1}-1\right)}\left(\xi_{1}\right) & \ldots & v_{1}^{\left(k_{n}-1\right)}\left(\xi_{n}\right) \\
\vdots & & \vdots & \vdots & & \vdots & & \vdots \\
0 & \ldots & 0 & v_{k}\left(\xi_{1}\right) & \ldots & v_{k}^{\left(k_{1}-1\right)}\left(\xi_{1}\right) & \ldots & v_{k}^{\left(k_{n}-1\right)}\left(\xi_{n}\right) \\
0 & \ldots & 0 & \psi\left(\xi_{1}\right) & \ldots & \psi^{\left(k_{1}-1\right)}\left(\xi_{1}\right) & \ldots & \psi^{\left(k_{n}-1\right)}\left(\xi_{n}\right)
\end{array}\right| \\
& =\left|\begin{array}{cccccccc}
u_{1,1} & \ldots & u_{1, m} & w_{1}\left(\xi_{1}\right) & \ldots & w_{1}^{\left(k_{1}-1\right)}\left(\xi_{1}\right) & \ldots & w_{1}^{\left(k_{n}-1\right)}\left(\xi_{n}\right) \\
\vdots & & \vdots & \vdots & & \vdots & & \vdots \\
u_{m+k, 1} & \ldots & u_{m+k, m} & w_{m+k}\left(\xi_{1}\right) & \ldots & w_{m+k}^{\left(k_{1}-1\right)}\left(\xi_{1}\right) & \ldots & w_{m+k}^{\left(k_{n}-1\right)}\left(\xi_{n}\right) \\
q_{1} & \ldots & q_{m} & g\left(\xi_{1}\right) & \ldots & g^{\left(k_{1}-1\right)}\left(\xi_{1}\right) & \ldots & g^{\left(k_{n}-1\right)}\left(\xi_{n}\right)
\end{array}\right|
\end{aligned}
$$

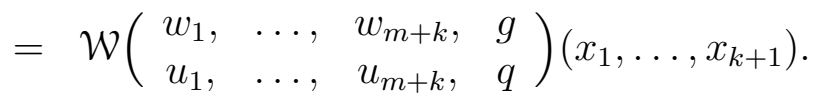

Thus, we have checked that the left hand side of (17) coincides with that of (4). The equality of the right hand sides follows similarly, and therefore, the proof is complete.

We now derive a useful consequence of Theorem 1.

Theorem 2. Let $I \subset \mathbb{R}$ be an interval and $[a, b]$ be a proper subinterval ofI. Let $1 \leq k, 1 \leq m$ be integers and $y_{1}, \ldots, y_{m} \in I \backslash[a, b]$. Assume that $w_{1}, \ldots, w_{m+k}: I \rightarrow \mathbb{R}$ are sufficently many times differentiable functions such that

$$
\mathcal{W}\left(w_{1}, \ldots, w_{m+n}\right)(y_{1}, \ldots, y_{m}, \underbrace{\xi, \ldots, \xi}_{n \text { times }}) \neq 0
$$

for all $\xi \in[a, b]$ and $n=0, \ldots, k$. Then, for all nonidentical points $x_{1}, \ldots, x_{k+1} \in[a, b]$, and functions sufficently many times differentiablef, $g: I \rightarrow \mathbb{R}$, there exists an intermediate point 
$\xi \in] \min x_{i}, \max x_{i}[$ such that

$$
\begin{aligned}
& \mathcal{W}\left(w_{1}, \ldots, w_{m+k}, f\right)(y_{1}, \ldots, y_{m}, \underbrace{\xi, \ldots, \xi}_{k+1 \text { times }}) \cdot \mathcal{W}\left(w_{1}, \ldots, w_{m+k}, g\right)\left(y_{1}, \ldots, y_{m}, x_{1}, \ldots, x_{k+1}\right) \\
& =\mathcal{W}\left(w_{1}, \ldots, w_{m+k}, g\right)(y_{1}, \ldots, y_{m}, \underbrace{\xi, \ldots, \xi}_{k+1 \text { times }}) \cdot \mathcal{W}\left(w_{1}, \ldots, w_{m+k}, f\right)\left(y_{1}, \ldots, y_{m}, x_{1}, \ldots, x_{k+1}\right) .
\end{aligned}
$$

Proof. Let $\pi$ be a permutation of the set $\{1, \ldots, m\}, \eta_{1}, \ldots, \eta_{l} \in I$, and $m_{1}, \ldots, m_{l} \in \mathbb{N}$ with $m_{1}+\cdots+m_{l}=m$ such that

$$
\left(y_{\pi(1)}, \ldots, y_{\pi(m)}\right)=(\underbrace{\eta_{1}, \ldots, \eta_{1}}_{m_{1} \text { times }}, \ldots, \underbrace{\eta_{l}, \ldots, \eta_{l}}_{m_{l} \text { times }}) .
$$

Set, for $i=1, \ldots, m+k$,

$$
u_{i}:=\left(u_{i 1}, \ldots, u_{i m}\right):=\left(w_{i}\left(\eta_{1}\right), \ldots, w_{i}^{\left(m_{1}-1\right)}\left(\eta_{1}\right), \quad \ldots, \quad w_{i}\left(\eta_{l}\right), \ldots, w_{i}^{\left(m_{l}-1\right)}\left(\eta_{l}\right)\right),
$$

and

$$
\begin{aligned}
& p:=\left(p_{1}, \ldots, p_{m}\right):=\left(f\left(\eta_{1}\right), \ldots, f^{\left(m_{1}-1\right)}\left(\eta_{1}\right), \ldots, \quad f\left(\eta_{l}\right), \ldots, f^{\left(m_{l}-1\right)}\left(\eta_{l}\right)\right), \\
& q:=\left(q_{1}, \ldots, q_{m}\right):=\left(g\left(\eta_{1}\right), \ldots, g^{\left(m_{1}-1\right)}\left(\eta_{1}\right), \quad \ldots, \quad g\left(\eta_{l}\right), \ldots, g^{\left(m_{l}-1\right)}\left(\eta_{l}\right)\right) .
\end{aligned}
$$

Observe, that with this notations, the conditions of Theorem 1 are satisfied and therefore there exists $\xi$ such that (4) holds. It is immediate to see that (4) is equivalent to (19).

\section{REFERENCES}

[AH79] G. Aumann and O. Haupt, Einführung in die reelle Analysis, Band II, W. de Gruyter, Berlin - New York, 1979. [HA38] O. Haupt and G. Aumann, Differential- und Integralrechnung, Band II, W. de Gruyter, Berlin - New York, 1938.

[Pal94] Zs. Páles, A unified form of the classical mean value theorems, in: Inequalities and Applications (ed. by R. P. Agarwal), World Scientific Publ., Singapore-New Jersey-London-Hong Kong, 1994, 493-500.

[RR87] J. Rätz and D. Russell, An extremal problem related to probability, Aequationes Math., 34 (1987), 316-324.

[Sch81] L. Schumaker, Spline Functions, Wiley, New York - Toronto, 1981.

Institute of Mathematics, University of Debrecen, H-4010 Debrecen, Pf. 12, Hungary

E-mail address: pales@science.unideb.hu 